\title{
Introduction to Special Issue "Human Enhancement Technologies and Our Merger with Machines"
}

\author{
Woodrow Barfield ${ }^{1, *}$ and Sayoko Blodgett-Ford ${ }^{2,3, *}$ \\ 1 Whitaker Institute, National University of Ireland, H91 TK33 Galway, Ireland \\ 2 Boston College Law School, Boston College, Newton Centre, MA 02459, USA \\ 3 GTC Law Group PC \& Affiliates, Westwood, MA 02090, USA \\ * Correspondence: Jbar5377@gmail.com (W.B.); blodgesa@bc.edu (S.B.-F.)
}

check for

updates

Citation: Barfield, W.; Blodgett-Ford, S. Introduction to Special Issue "Human Enhancement Technologies and Our Merger with Machines".

Philosophies 2021, 6, 9.

https://doi.org/10.3390/

philosophies6010009

Received: 22 January 2021

Accepted: 25 January 2021

Published: 29 January 2021

Publisher's Note: MDPI stays neutral with regard to jurisdictional claims in published maps and institutional affiliations.

Copyright: (c) 2021 by the authors. Licensee MDPI, Basel, Switzerland. This article is an open access article distributed under the terms and conditions of the Creative Commons Attribution (CC BY) license (https:// creativecommons.org/licenses/by/ $4.0 /)$.
We are pleased to introduce the authors and papers which form the Special Issue "Human Enhancement Technologies and Our Merger with Machines". We should first note that humans are already becoming equipped with technology that ranges from artificial limbs, heart pacers, retinal and neuroprostheses, cochlear implants, and other emerging technologies used to enhance human capabilities. We had such current and future "mechanical enhancements" in mind when we first considered editing a Special Issue. However, as we developed the Special Issue further, based on the authors submissions in response to the call for papers, we realized that it was essential to develop a broad definition of "enhancement" to include chemical enhancements, genetic enhancements, pharmaceutical enhancements, and beyond, as all such areas are currently under development and relate to the topic of the Special Issue. In our view, a Special Issue on human enhancement technologies is not only timely but covers a topic that invokes many interesting and challenging ethical, legal, practical, and philosophical issues. When inviting scholars to contribute to the Special Issue, we sought authors from different academic disciplines such that we would produce a Special Issue with different perspectives and points of view. We think a cross-disciplinary approach to human enhancement technologies is important given the complexity and depth of the challenges that need to be addressed. Thus, the Special Issue consists of eight papers contributed by a diverse group of scholars from law, technology, and social science that focus on issues which involve a future in which people will be equipped with enhancement technologies for medical and nonmedical reasons.

The paper by Dunagan, Grove, and Halbert, "The Neuropolitics of Brain Science and Its Implications for Human Enhancement and Intellectual Property Law", focuses on how human enhancements directed at the brain may change what we know about human creativity and innovation. The authors make the points that the possibility of direct brain-to-brain communication, the use of cognitive-enhancing drugs to enhance intelligence and creativity, and the extended connections between brains and the larger technological world can lead to a linkage between intellectual property (IP) law and policy. To address IP issues resulting from human enhancements, the authors suggest that new conceptualizations of the brain are needed to challenge the notion of the autonomous individual and to address creativity and originality as possibly occurring beyond that of an individual creation. Fiorella Battaglia, in "Agency, Responsibility, Selves, and the Mechanical Mind", also focuses on the subject of mental activities and human enhancement, discussing moral issues that arise not only when neural technology directly influences and affects people's lives, but also when the impact of its interventions in creating an "extended mind" allows us to conceptualize the mind in new and unexpected ways, using a "mechanical mind" approach. By reviewing theories of consciousness, theories of subjectivity, and third person perspective on the brain, the paper identifies both a major area of transformation in philosophy of action understood in terms of additional epistemic devices and as a result "self-objectification" as a new area of concern, which she characterizes in terms of "alienation" following Ernst Kapp's philosophy of technology. 
Allen Coin, Megan Mulder, and Veljko Dublievic, in "Ethical Aspects of BCI Technology: What Is the State of the Art?", address uses of enhancement technology to discuss how brain activity can be interpreted through both invasive and noninvasive monitoring devices, allowing for novel, therapeutic solutions for individuals with disabilities and for other nonmedical applications. However, the authors note that, to date, a number of ethical issues have been identified from the use of brain-computer interface (BCI) technology. Based on a review and analysis of the literature, the authors identify key areas of $\mathrm{BCI}$ ethics that they argue warrant further research efforts, arguing that the physical and psychological effects of $\mathrm{BCI}$ technology and, particularly, BCI ethics should be addressed in future work. From more of a technological view of human enhancement, Kevin Warwick in "Superhuman Enhancements via Implants: Beyond the Human Mind" takes a practical look, based on his unique perspective of one of the world's first cyborgs, at some of the possible enhancements for humans through the use of implants, particularly in the brain or nervous system. An interesting point is made by Warwick that some cognitive enhancements may not turn out to be practically useful, whereas others may turn out to be mere steps on the way to the construction of superhumans. Warwick's paper focuses on the latter issue-enhancements that take recipients beyond the human norm rather than any implantations employed merely for therapy. Warwick takes the perspective that humans are essentially their brains, and that bodies serve as interfaces between brains and the environment. Speculating about the future of human enhancement, Warwick considers the possibility of building an "Interplanetary Creature" having an intelligence and, possibly, a consciousness of its own.

Chia Wei Fahn, in "Marketing the Prosthesis: Supercrip and Superhuman Narratives in Contemporary Cultural Representations", examines prosthetic technology in the context of posthumanism and disability studies, with a focus on how the disabled body is empowered through prosthetic enhancement and cultural representations. Fahn cross-examines empowering marketing images and phrases embedded in cinema and media that emphasize how disability becomes a superability with prosthetic enhancement. She discusses questions such as: Why is it that the disabled have yet to reap the rewards or prosthetic technology? How are disabled bodies, biotechnology, and posthuman possibilities commodified and commercialized? Most importantly, what impact will human enhancements have on our society? Presenting a different but important type of enhancement technology, Marcelo de Araujo in "The Ethics of Genetic Cognitive Enhancement: Gene Editing or Embryo Selection?" discusses how recent research with human embryos in different parts of the world has sparked a new debate on the ethics of genetic human enhancement. While focusing on gene-editing technologies, especially CRISPR, Araujo notes that less attention has been given to the prospect of pursuing genetic human enhancement by means of in vitro fertilization (IVF) in conjunction with in vitro gametogenesis, genome-wide association studies, and embryo selection. Thus, he examines the different ethical implications of the quest for cognitive enhancement by means of gene-editing on the one hand, and embryo selection on the other. He argues that the philosophical debate on the ethics of enhancement should take into consideration public attitudes to research on human genomics and human enhancement technologies.

S.J. Blodgett-Ford, in "Human Enhancements and Voting: Towards a Declaration of Rights and Responsibilities of Beings", explores the ethics of "voting" in the context of human enhancements. The author discusses "voting" for enhanced humans who fall into two broad categories - those with moderate enhancements, where the "humanity" of the enhanced person is not seriously in question, and those with extreme enhancements, where humans who lacked similar enhancements would likely question the "humanity" of the enhanced human (people a hundred years ago might think enhanced people of today are beyond humanity). The question of who has the right to vote and how they can vote is explored in the context of voting in the United States at the federal level. The author points out that for voting, there is risk that existing patterns of discrimination will continue substantially "as is" for humans with physical and mental enhancements, 
who are viewed as "still human". For the "beyond human" category of enhancements, the article argues that the established rules and practice of voting are likely to be directly challenged by extreme physical and/or mental enhancements, and that for both moderate and extreme enhancements, humans who are not enhanced may be disenfranchised if certain enhancements become prevalent among the voting population. Extending the discussion, the author borrows from the Universal Declaration of Human Rights (UDHR) of 1948 and applies an extended ethical theory of engagement to advocate that voting rights and responsibilities should be reframed from a foundational working hypothesis that all living and all conscious "beings" (including all enhanced and nonenhanced humans) are of value and should have a right to vote as a cornerstone of a new Declaration of Rights and Responsibilities of Beings.

Given the use of enhancements technologies to create "super soldiers", Sahar Latheef and Adam Henschke in "Can a Soldier Say No to an Enhancing Intervention?" discuss how technological advancements have provided militaries with the possibility to enhance human performance and to provide soldiers with better warfighting capabilities. Though these technologies hold significant potential, their use is not without cost to the individual. The paper explores the complexities associated with using human cognitive enhancements in the military, focusing on how the purpose and context of these technologies could potentially undermine a soldier's ability to say no to these interventions. The authors explore situations that could potentially compel a soldier to accept such technologies and how this acceptance could impact rights to individual autonomy and informed consent within the military. The authors argue that though in some situations, a soldier may be compelled to accept enhancements, with their right to say no diminished, it is not a blanket rule, and safeguards ought to be in place to ensure that autonomy and informed consent are not overridden.

To conclude, due to the importance of the ethical and other philosophical, legal, social, and scientific issues involved in human enhancement technologies (broadly defined), we hope that the Special Issue generates more discussion and research on the topics covered in the eight papers and beyond. In our view, a cross-disciplinary approach within the fields of philosophy, engineering, computer science, law, and social science is critical to approach the involved ethical issues as increasingly more powerful technology is attached to and implanted within the human body, and as other modifications become possible.

Funding: This research received no external funding.

Acknowledgments: The editors gratefully acknowledge the efforts of the contributors to the special edition and the quality of their papers.

Conflicts of Interest: The authors declare no conflict of interest. 